\title{
Review \\ Potential of Intestinal Current Measurement for Personalized Treatment of Patients with Cystic Fibrosis
}

\author{
Simon Y. Graeber ${ }^{1,2,3} \mathbb{D}^{\text {, Constanze Vitzthum }}{ }^{1,3}$ and Marcus A. Mall ${ }^{1,2,3, *(1)}$ \\ 1 Charité-Universitätsmedizin Berlin, Department of Pediatric Respiratory Medicine, \\ Immunology and Critical Care Medicine, 13353 Berlin, Germany; simon.graeber@charite.de (S.Y.G.); \\ constanze.vitzthum@charite.de (C.V.) \\ 2 Berlin Institute of Health, Charité-Universitätsmedizin Berlin, 10117 Berlin, Germany \\ 3 German Centre for Lung Research (DZL), 13353 Berlin, Germany \\ * Correspondence: marcus.mall@charite.de; Tel.: +49-(30)-450-566-182; Fax: +49-(30)-450-566-931
}

Citation: Graeber, S.Y.; Vitzthum, C.; Mall, M.A. Potential of Intestinal Current Measurement for Personalized Treatment of Patients with Cystic Fibrosis. J. Pers. Med. 2021, 11, 384. https://doi.org/ 10.3390/jpm11050384

Academic Editors: Cornelis K. van der Ent and Harry G. M. Heijerman

Received: 19 April 2021

Accepted: 4 May 2021

Published: 8 May 2021

Publisher's Note: MDPI stays neutral with regard to jurisdictional claims in published maps and institutional affiliations.

Copyright: (c) 2021 by the authors. Licensee MDPI, Basel, Switzerland. This article is an open access article distributed under the terms and conditions of the Creative Commons Attribution (CC BY) license (https:// creativecommons.org/licenses/by/ $4.0 /)$.

\begin{abstract}
Refinement of personalized treatment of cystic fibrosis (CF) with emerging medicines targeting the CF basic defect will likely benefit from biomarkers sensitive to detect improvement of cystic fibrosis transmembrane conductance regulator (CFTR) function in individual patients. Intestinal current measurement (ICM) is a technique that enables quantitative assessment of CFTR chloride channel function in rectal tissues or other intestinal epithelia. ICM was originally developed to study the CF ion transport defect in the intestine and has been established as a sensitive biomarker of CFTR function and diagnostic test for CF. With the emergence of CFTR-directed therapeutics, ICM has become an important tool to estimate the level of rescue of CFTR function achieved by approved CFTR modulators, both at the level of CFTR genotype groups, as well as individual patients with CF. In combination with preclinical patient-derived cell culture models, ICM may aid the development of targeted therapies for patients with rare CFTR mutations. Here, we review the principles of ICM and examine how this CFTR biomarker may be used to support diagnostic testing and enhance personalized medicine for individual patients with common as well as rare CFTR mutations in the new era of medicines targeting the underlying cause of CF.
\end{abstract}

Keywords: cystic fibrosis; CFTR; intestinal current measurement (ICM); personalized medicine

\section{Introduction}

Recent breakthroughs in cystic fibrosis (CF) transmembrane conductance regulator (CFTR)-directed therapies have heralded a new era of precision medicine for patients with CF [1-13]. Biomarkers that are sensitive to detect improvement of CFTR function in individual patients could help to assess individual treatment responses to these therapies targeting the underlying CF defect. In addition to sweat chloride concentration as a measurement of CFTR function in the sweat duct, intestinal current measurement (ICM) has been developed as a technique that enables quantitative assessment of CFTR chloride channel function in native intestinal tissues. Shortly after the discovery of the CFTR gene in 1989 [14], several research groups developed modified Ussing chambers for transepithelial measurements across native tissues from the rectum and other regions of the intestinal mucosa to study the CF ion transport defect in the intestine [15-31]. At this time, the focus was on understanding the role of CFTR as a cAMP-dependent chloride channel in ion and fluid transport in the gastro-intestinal tract, and the effect of CFTR dysfunction caused by mutations in the CFTR gene on this process. In these studies it was found that, in contrast to the airways where CFTR is expressed together with the alternative calcium-activated chloride channel TMEM16A that remains intact in CF [32-39], CFTR is the dominant chloride channel responsible for chloride and fluid secretion in human colon $[18,21,22,24,27,30]$. The characterization of CFTR dysfunction in these studies led to the development of experimental protocols that were able to differentiate between 
impaired CFTR function in intestinal tissues from patients with CF vs. normal CFTR function in control subjects $[29,31]$. In addition, protocols were developed that enabled the classification of CF tissues into two groups with (i) lack of or minimal detectable CFTR function and (ii) residual CFTR function [28,29,31,40]. Based on these results, ICM was established as a diagnostic test to aid in establishing or refuting a diagnosis of $\mathrm{CF}$, if sweat test results are equivocal and/or if the functional consequence of rare or newly detected CFTR variants are unknown. With the emergence of CFTR-directed therapeutics in the clinical arena, sensitive biomarkers of CFTR function such as ICM have the potential to facilitate and enhance personalized therapy for patients with CF [1,2,41,42]. In this context, ICM was shown to be sensitive to detect improvement of CFTR function when intestinal tissue biopsies were treated with CFTR modulators ex vivo or obtained from patients with CF that were treated systemically with CFTR modulators [43-46]. In this review, we describe the principles of ICM, summarize its use as a sensitive biomarker of CFTR function, and examine recent studies using ICM as an outcome measure of response to CFTR-directed therapeutics, and how the use of ICM may contribute to further improvement in personalized medicine eventually for all patients with $\mathrm{CF}$.

\section{Principle of Intestinal Current Measurement (ICM)}

For ICM, small superficial biopsies of the rectal mucosa (i.e., only epithelial layer) are obtained by forceps or suction biopsy [21,29,31]. Collection of the biopsies is painless, does not require sedation and is safe and easy to perform at any age. Rectal tissues are immediately mounted in perfused or circulating small aperture Ussing chambers and equilibrated in physiological buffer solution [21,29,31]. After equilibration, basal bioelectric properties are recorded under open-circuit or short-circuit conditions, and the effects of pharmacological stimuli on intestinal ion transport can be studied ex vivo in a controlled setting. Initially, two different setups of micro-Ussing chambers were developed to measure transepithelial currents after pharmacological activation of CFTR in native rectal tissues. The setup originally developed at the University Hospital of Rotterdam, The Netherlands, uses traditional re-circulating micro-Ussing chambers with a pharmacological protocol focusing mainly on calcium-mediated activation of the tissues to induce chloride secretion via CFTR $[18,19,26,31]$. The other setup, originally developed at the University of Freiburg, Germany, uses continuously perfused micro-Ussing chambers that enable studying the effects of pharmacological modulation of CFTR function under different experimental conditions (e.g., in the absence and presence of endogenous CFTR activation) in the same tissue in a strictly paired fashion. Pharmacological protocols with this setup enable the sequential assessment of cAMP-mediated and calcium-mediated chloride secretion as a readout of CFTR function $[21,24,27,29,30,47,48]$. Typically, ICM is performed in the presence of amiloride to block epithelial sodium channel (ENaC)-mediated sodium absorption and indomethacin to inhibit cyclooxygenase activity and, thus, suppress the synthesis of prostaglandins that stimulates endogenous cAMP formation in intestinal tissues [22,24].

Compared to airway tissues, rectal biopsies can be easily obtained by minimally invasive procedures and rectal tissue has several advantages for studies of CFTR function. First, rectal tissues express higher levels of CFTR resulting in a high signal-to-noise ratio. Second, in contrast to the airways, the rectal epithelium does not express alternative calcium-activated chloride channels [21,27], and, therefore, both cAMP- and calciummediated chloride secretion are strictly related to CFTR function. Finally, the intestine including the rectum is not affected by chronic inflammation, infection with CF pathogens or structural organ damage and remodeling, i.e., factors that may impede CFTR chloride channel function independent of the basic molecular defect of CFTR mutations. 


\section{ICM as a Biomarker of CFTR Function and Diagnostic Test for CF}

Based on the results of the early studies on the pathophysiology of the CF ion transport defect in rectal tissue, standardized ICM protocols for pharmacological stimulation of CFTR chloride channels were established. These protocols were shown to differentiate between normal CFTR function in control subjects and a spectrum of CFTR dysfunction in CF, ranging from the complete absence of CFTR-mediated chloride secretion to substantial residual CFTR function detected by ICM in patients with different CFTR genotypes [28,40,47,49]. Specifically, it was found that in rectal tissues from healthy individuals, cAMP-dependent activation induces a sustained CFTR-mediated chloride secretory response [21]. This chloride secretory response is augmented by cholinergic co-activation with carbachol via activation of calcium-dependent potassium channels in the basolateral membrane of colonocytes that leads to an increase in the driving force for CFTR-mediated chloride secretion across the luminal membrane [37]. In patients with CF, the responses detected by ICM allow to distinguish between two functional phenotypes. In the majority of patients $(\sim 85 \%)$, CFTR-mediated chloride secretory responses are absent and instead ICM shows an inverse bioelectric response following cAMP- and calcium-dependent stimulation that was found to reflect luminal potassium secretion [24]. In a series of studies, it was shown that CF patients with this ICM functional signature typically carry two 'severe' CFTR mutations (i.e., class I to III) $[28,40,47,50]$. Further, it was shown that this absence of CFTR function determined by ICM is associated with a classical CF phenotype including sweat chloride concentrations in the upper diagnostic range, progressive sino-pulmonary disease and exocrine pancreatic insufficiency $[28,29,40,47,50]$. In the remaining group of patients $(\sim 15 \%)$, ICM detects residual CFTR function, as evidenced by an attenuated chloride secretory response to cAMP-dependent stimulation, as well as cholinergic co-activation typically reflected by a biphasic response upon co-stimulation of rectal tissues with carbachol $[28,47]$. Studies on the relationship between CFTR function detected by ICM, CFTR genotype and CF phenotype demonstrated that patients in this group typically carry at least one 'mild' CFTR mutation (class IV and V) and that on average, residual CFTR function was associated with a milder form of $\mathrm{CF}$ characterized by sweat chloride concentrations in the lower diagnostic or intermediate range, long-term exocrine pancreatic sufficiency, later age at diagnosis, and less severe impairment in nutritional outcomes and lung function [28,51]. These studies support a prognostic value of ICM and led to the implementation of ICM in the diagnostic algorithm for $\mathrm{CF}$, especially to aid establish or refute a diagnosis of $\mathrm{CF}$ in patients with equivocal sweat test or genetic testing results [52]. In this context, recent studies also demonstrated the potential usefulness of ICM in the diagnosis of atypical CF and CFTR related disease [53,54]. In the future, with the widespread implementation of CF newborn screening in many countries, ICM may also become a useful tool for the diagnostic workup of CF newborn screening positive infants with inconclusive diagnosis (CF-SPID) to determine disease liability and prognosis [52,55-58]. Collectively, these studies have established ICM as a sensitive biomarker of CFTR function in the intestinal epithelium. However, due to the requirement of specialized technical equipment and well-trained personnel, availability of ICM remains limited to reference centers.

\section{ICM as Outcome Measure of In Vivo Response to CFTR-Directed Therapeutics}

The development and recent approval of an increasing number of CFTR modulator drugs has created an unprecedented opportunity to treat the basic defect in a growing number of patients with CF [4-13]. However, clinical trials of CFTR modulators showed heterogeneous responses in clinical outcomes as well as sweat chloride concentration among patients with identical CFTR genotypes. These results suggest that additional sensitive biomarkers of CFTR function will be important to study the degree of functional rescue of CFTR mutations by different CFTR modulator drugs, both at the level of CFTR genotype groups as well as individual patients. We expect these studies will be instrumental to determine response to therapy at the level of the CF basic defect independent of disease severity and/or environmental factors that may confound complex clinical outcomes such 
as spirometry $\left(\mathrm{FEV}_{1} \%\right.$ predicted), and thereby enhance the potential of personalized therapy for $\mathrm{CF}$.

In this context, initial 'real world' studies with approved CFTR modulators demonstrated that ICM is sensitive to detect in vivo activation of CFTR in individual CF patients with a G551D mutation treated with the CFTR potentiator ivacaftor [44]. Here, ICM detected activation of CFTR-mediated chloride secretion to a mean level of $~ 50 \%$ of normal in G551D CF patients after starting treatment with ivacaftor [44]. Further, ICM demonstrated that the combination of the CFTR corrector lumacaftor with the potentiator ivacaftor as the first CFTR modulator combination therapy approved for the treatment of CF patients homozygous for the common F508del mutation leads to partial rescue of CFTR activity to levels of $\sim 15-20 \%$ of normal CFTR function in healthy controls $[45,46]$. Interestingly, improvement in CFTR function detected by ICM was also observed in patients that did not show improvement in $\mathrm{FEV}_{1}$ \% predicted after initiation of lumacaftor-ivacaftor therapy [45]. In this study, ICM detected robust improvement of CFTR function in almost all patients and the variance of ICM was substantially lower than that of nasal potential difference (NPD; total chloride secretory response) and lung function measures [45]. These findings indicate that clinical outcome measures such as $\mathrm{FEV}_{1} \%$ predicted that are commonly used as endpoints in clinical trials have limitations as outcome measure of response to therapy at the level of the CF basic defect, and highlight the importance of including sensitive biomarkers of CFTR function in an approach to personalized medicine of CF. As the number of approved CFTR modulator drugs containing different combinations of small molecule compounds continues to increase, even for patients with the same CFTR genotype, ICM may become a useful tool for quantitative comparison of efficacy of functional correction of CFTR mutations at the level of genotype groups, and potentially serve as a biomarker to aid optimization of personalized treatment of individual patients.

\section{Potential Use of ICM for Personalized Medicine for Patients with Rare Mutations and High Unmet Need}

Almost a decade ago, the potentiator ivacaftor was the first approved CFTR modulator for patients with CF with a G551D gating mutation [4]. It was shown that ivacaftor restores CFTR function to $~ 50 \%$ of wild-type levels and improved FEV1 \% predicted by $\sim 10 \%$ in patients with at least one G551D mutation [4,44]. In patients with the common F508del mutation or other processing (class II) mutations, ivacaftor alone failed to improve CFTR function or lung function. However, the combination of a corrector (lumacaftor or tezacaftor), which partially overcomes the folding defect of F508del CFTR with the potentiator ivacaftor led to an improvement in CFTR function and clinical outcomes in patients homozygous for the F508del mutation $[5,6]$. Recently, a triple combination of the two correctors elexacaftor and tezacaftor with the potentiator ivacaftor showed substantial clinical improvement in patients with CF with at least one F508del allele [10,11]. As a result, effective CFTR modulators have recently been approved and are becoming available for the treatment of approximately $90 \%$ of CFTR genotypes [4-13]. Despite this unprecedented breakthrough in precision medicine of the underlying $\mathrm{CF}$ defect, there are still approximately $10 \%$ of patients with CFTR genotypes that cannot be treated with current CFTR-directed therapeutics. Therefore, preclinical development and testing of new therapies targeting rare CFTR mutations that do not respond to current CFTR modulators, or for which responsiveness remains unknown due to their low occurrence, remains important to further improve precision medicine and ultimately provide effective therapies for all patients with CF. In this context, ICM was shown to detect improvement in CFTRmediated chloride secretion in response to stimulation with a CFTR potentiator in rectal tissue of patients with a broad range of residual CFTR function mutations (Y161C, V232D, R334W, T338I, I1234V, 3272-26 A > G, $3849+10 \mathrm{~kb}$ C > T, $4005+5727$ A > G, G576A, F1052V, M1137R, $1898+3 \mathrm{~A}>\mathrm{G}$ ) [43]. These studies also showed that ex vivo testing of multiple compounds and compound combinations in freshly excised rectal tissue by ICM is limited by the number of rectal biopsies that can be obtained as well as their limited viability even under primary tissue culture conditions. However, in addition to ICM, rectal 
biopsies can also be used to generate patient-derived intestinal organoids as a versatile model system for preclinical testing of investigational compounds targeting rare CFTR mutations that are not eligible for treatment with an approved CFTR modulator [59,60]. In addition, nasal or bronchial epithelial cells collected by brushings can be expanded and differentiated under air-liquid interface conditions to enable testing of multiple drug candidates in primary airway epithelial cultures derived from individual patients harboring specific CFTR mutations [61-70]. Several preclinical studies in these patient-derived model systems showed that the triple combination of elexacaftor-tezacaftor-ivacaftor, as well as several novel modulator combinations improve CFTR function in class II mutations other than F508del, as well as other rare CFTR mutations [68-74]. Functional in vitro assays in these patient-derived intestinal and airway model systems were shown to correlate with biomarkers of CFTR function in patients with a spectrum of CFTR mutations associated with a broad range of CFTR modulator responses [62,75]. However, recent studies found no correlation between the magnitude of in vitro response in rectal organoids and in vivo response to CFTR modulator treatment detected by measurements of sweat chloride concentration, NPD or ICM in more homogenous patient groups receiving the same CFTR modulator $[76,77]$. These findings indicate that in vivo restoration of mutant CFTR function probably depends on multiple factors including pharmacokinetics that cannot be entirely predicted by these patient-derived in vitro models. Therefore, biomarkers of in vivo CFTR function remain important for the assessment of response to therapy also in individual patients, which can be achieved once the investigational compound can be administered to patients. Among the different in vivo biomarkers of CFTR function, sweat chloride concentration is the most broadly available and was shown to detect improvement even in F508del homozygous patients treated with lumacaftor-ivacaftor with moderate improvement of CFTR function [45,78,79]. However, in about 20\% of F508del homozygous patients, improvement in sweat chloride concentration under treatment with lumacaftorivacaftor did not correspond with improvement of CFTR function detected by ICM or NPD [45]. These results suggest that ICM and NPD can capture additional, potentially organ-specific aspects of in vivo rescue of mutant CFTR function by CFTR modulator therapy. On note, in studies comparing NPD and ICM as in vivo biomarkers of CFTR function, ICM was superior to NPD in distinguishing between patients with CF and healthy controls, and ICM demonstrated substantially greater power than NPD to detect low levels of residual CFTR function $[50,80]$. In sample size calculations based on this study, as little as five patients may be sufficient to detect a ten percent improvement of CFTR function by ICM, highlighting the high sensitivity of ICM as well as its potential in early phase clinical trials [50]. Along these lines, in our previous real world study evaluating the effects of lumacaftor-ivacaftor in F508del homozygous patients with CF, we found that ICM detected improvement in CFTR function in $95 \%$ of patients compared to $65 \%$ of patients showing functional improvement in response to therapy by NPD [45]. Taken together, these results support ICM as a sensitive outcome measure for testing of in vivo efficacy of CFTR modulators. In combination with patient-derived in vitro cell culture models for preclinical testing of investigational compounds ICM may therefore facilitate the development of effective therapies of the basic defect for all patients with CF (Figure 1). 


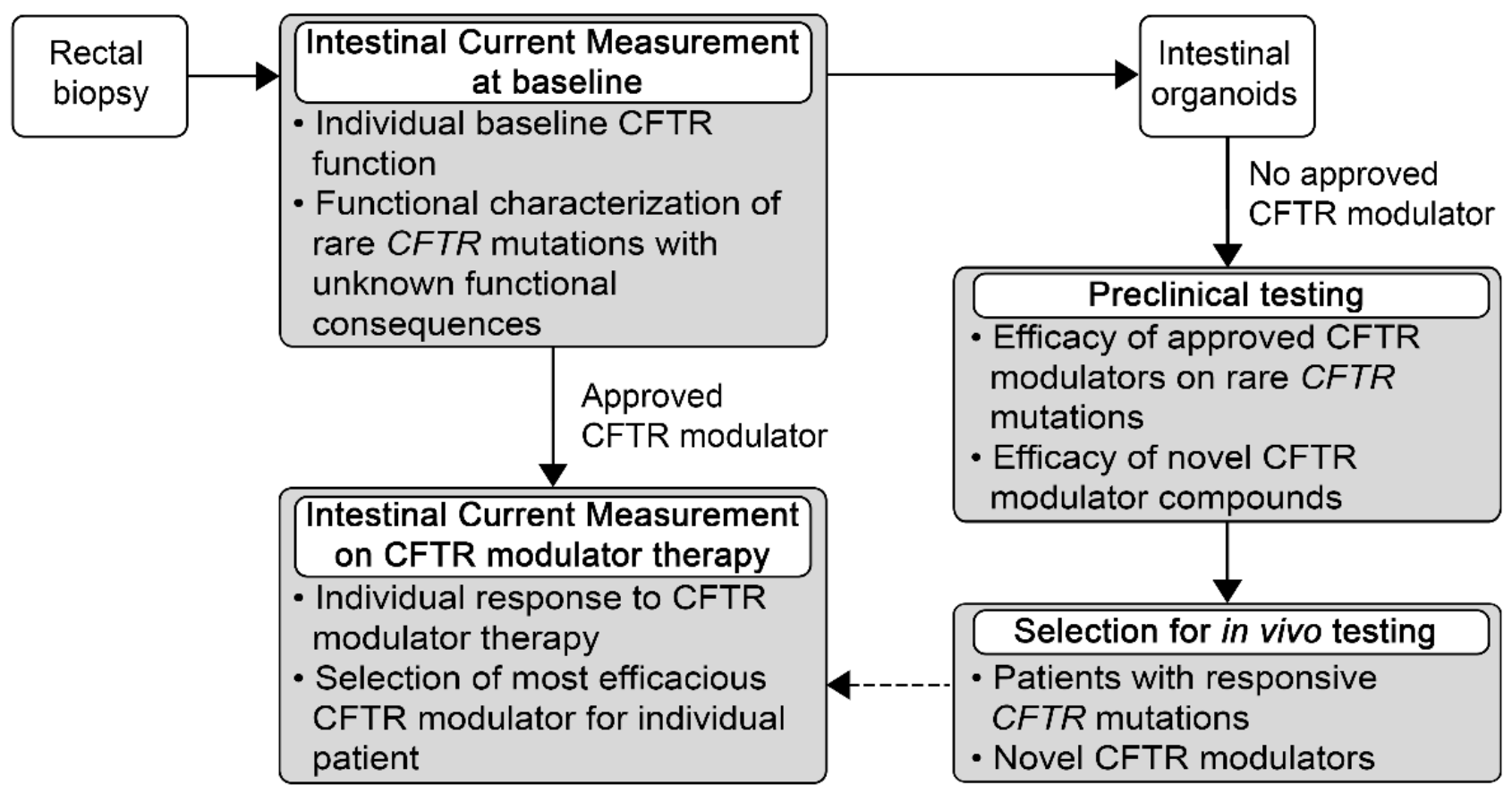

Figure 1. Use of intestinal current measurement for personalized medicine of CF. Intestinal current measurement (ICM) in rectal biopsies can determine individual baseline cystic fibrosis (CF) transmembrane conductance regulator (CFTR) function and provide functional characterization of rare mutations with unknown functional consequences. In CF patients with CFTR genotypes that can be treated with approved CFTR modulators, ICM can be used to determine the individual response to therapy at the level of the CF basic defect. If multiple approved CFTR modulator therapies are available, ICM can help to find the most efficacious drug for the individual patient. However, as ICM detects the improvement of in vivo CFTR function, patients needed to be treated with the different CFTR modulator therapies and repeated rectal biopsies would be necessary to compare responses to these different drug regimen. In patients with rare CFTR mutations and genotypes with no approved CFTR modulator therapies, rectal biopsies obtained for ICM can be used to generate intestinal organoids for preclinical testing of (i) responsiveness of rare mutations to approved CFTR modulators; and (ii) evaluation of promising novel compounds. ICM may help to verify results from preclinical studies in n-of-1 studies or early phase clinical trials.

\section{Conclusions}

In summary, ICM is a valuable biomarker to characterize CFTR function in native intestinal tissues and a sensitive outcome measure of in vivo response to CFTR modulator therapy in patients with CF (Table 1). In patients with access to CFTR modulator therapies, ICM can therefore be used to determine the level of functional rescue, both at the level of genotype groups as well as individual patients. As an increasing number of CFTR modulators is becoming available for patients with common CFTR mutations, ICM may help to select the most efficacious available CFTR modulator for the individual patient. For patients with rare CFTR mutations with unknown functional consequences and no access to CFTR modulator treatment, functional characterization with ICM may help to identify potential treatment options. Further, rectal tissue collected for ICM can also be used for the generation of intestinal organoids for preclinical in vitro testing of novel drug candidates. Promising candidates may then be tested using ICM as sensitive CFTR biomarker to determine in vivo efficacy in early phase proof-of-concept clinical trials or n-of- 1 studies especially for patients with rare CFTR mutations. We conclude that ICM, as a sensitive CFTR biomarker for quantitative assessment of response to CFTR-directed therapeutics at the level of the CF basic defect, has a high potential to facilitate enhancement of personalized medicine for patients with common as well as rare CFTR genotypes. 
Table 1. Published studies using intestinal current measurement (ICM) as a biomarker of cystic fibrosis transmembrane conductance regulator (CFTR) function.

\begin{tabular}{cc}
\hline Category & Reference \\
\hline Development of ICM & {$[15-19,21,22,24,26]$} \\
ICM as a diagnostic test for CF & {$[28-31,40,42,47,49,50,53,54,75,80]$} \\
$\begin{array}{c}\text { ICM as outcome measure of preclinical ex vivo } \\
\text { response to CFTR-directed therapeutics } \\
\text { ICM as outcome measure of in vivo response } \\
\text { to CFTR-directed therapeutics }\end{array}$ & {$[25,43,48]$} \\
\hline
\end{tabular}

Author Contributions: Conception and design of the review: S.Y.G., M.A.M.; acquisition, analysis and interpretation of the literature: S.Y.G., C.V., M.A.M.; drafting the article or revising it critically for important intellectual content: S.Y.G., C.V., M.A.M. All authors have read and agreed to the published version of the manuscript.

Funding: This work was supported in part by grants from the German Federal Ministry of Education and Research (82DZL009B1) and the Einstein Foundation Berlin (EP-2017-393). The funders had no role in the writing of the manuscript, or in the decision to publish this review.

Acknowledgments: S.Y.G. is participant in the BIH-Charité Clinician Scientist Program funded by the Charité-Universitätsmedizin Berlin and the Berlin Institute of Health. We acknowledge support from the German Research Foundation (DFG) and the Open Access Publication Fund of Charité -Universitätsmedizin Berlin. The authors thank their colleagues who contributed to the work cited in this review.

Conflicts of Interest: S.Y.G. reports fees for advisory board participation from Chiesi outside the submitted work. M.A.M. reports research grants and patient recruitment fees for clinical trials from Vertex, for which his institution Charité-Universitätsmedizin Berlin received payment; fees for consulting and advisory board participation from Antabio, Arrowhead, Boehringer Ingelheim, Enterprise Therapeutics, Kither Biotech, Santhera, Sterna Biologicals, and Vertex Pharmaceuticals outside the submitted work.

\section{References}

1. Mall, M.A.; Mayer-Hamblett, N.; Rowe, S.M. Cystic Fibrosis: Emergence of Highly Effective Targeted Therapeutics and Potential Clinical Implications. Am. J. Respir. Crit. Care Med. 2020, 201, 1193-1208. [CrossRef] [PubMed]

2. Bell, S.C.; Mall, M.A.; Gutierrez, H.; Macek, M.; Madge, S.; Davies, J.C.; Burgel, P.R.; Tullis, E.; Castanos, C.; Castellani, C.; et al. The future of cystic fibrosis care: A global perspective. Lancet Respir. Med. 2020, 8, 65-124. [CrossRef]

3. Gentzsch, M.; Mall, M.A. Ion Channel Modulators in Cystic Fibrosis. Chest 2018, 154, 383-393. [CrossRef]

4. Ramsey, B.W.; Davies, J.; McElvaney, N.G.; Tullis, E.; Bell, S.C.; Drevinek, P.; Griese, M.; McKone, E.F.; Wainwright, C.E.; Konstan, M.W.; et al. A CFTR potentiator in patients with cystic fibrosis and the G551D mutation. N. Engl. J. Med. 2011, 365, 1663-1672. [CrossRef]

5. Wainwright, C.E.; Elborn, J.S.; Ramsey, B.W.; Marigowda, G.; Huang, X.; Cipolli, M.; Colombo, C.; Davies, J.C.; de Boeck, K.; Flume, P.A.; et al. Lumacaftor-Ivacaftor in Patients with Cystic Fibrosis Homozygous for Phe508del CFTR. N. Engl. J. Med. 2015, 373, 220-231. [CrossRef] [PubMed]

6. Taylor-Cousar, J.L.; Munck, A.; McKone, E.F.; van der Ent, C.K.; Moeller, A.; Simard, C.; Wang, L.T.; Ingenito, E.P.; McKee, C.; Lu, Y.; et al. Tezacaftor-Ivacaftor in Patients with Cystic Fibrosis Homozygous for Phe508del. N. Engl. J. Med. 2017, 377, $2013-2023$. [CrossRef] [PubMed]

7. Rowe, S.M.; Daines, C.; Ringshausen, F.C.; Kerem, E.; Wilson, J.; Tullis, E.; Nair, N.; Simard, C.; Han, L.; Ingenito, E.P.; et al. Tezacaftor-Ivacaftor in Residual-Function Heterozygotes with Cystic Fibrosis. N. Engl. J. Med. 2017, 377, 2024-2035. [CrossRef]

8. Keating, D.; Marigowda, G.; Burr, L.; Daines, C.; Mall, M.A.; McKone, E.F.; Ramsey, B.W.; Rowe, S.M.; Sass, L.A.; Tullis, E.; et al. VX-445-Tezacaftor-Ivacaftor in Patients with Cystic Fibrosis and One or Two Phe508del Alleles. N. Engl. J. Med. 2018, 379, 1612-1620. [CrossRef]

9. Davies, J.C.; Moskowitz, S.M.; Brown, C.; Horsley, A.; Mall, M.A.; McKone, E.F.; Plant, B.J.; Prais, D.; Ramsey, B.W.; Taylor-Cousar, J.L.; et al. VX-659-Tezacaftor-Ivacaftor in Patients with Cystic Fibrosis and One or Two Phe508del Alleles. N. Engl. J. Med. 2018, 379, 1599-1611. [CrossRef]

10. Middleton, P.G.; Mall, M.A.; Drevinek, P.; Lands, L.C.; McKone, E.F.; Polineni, D.; Ramsey, B.W.; Taylor-Cousar, J.L.; Tullis, E.; Vermeulen, F.; et al. Elexacaftor-Tezacaftor-Ivacaftor for Cystic Fibrosis with a Single Phe508del Allele. N. Engl. J. Med. 2019, 381, 1809-1819. [CrossRef] 
11. Heijerman, H.G.M.; McKone, E.F.; Downey, D.G.; Van Braeckel, E.; Rowe, S.M.; Tullis, E.; Mall, M.A.; Welter, J.J.; Ramsey, B.W.; McKee, C.M.; et al. Efficacy and safety of the elexacaftor plus tezacaftor plus ivacaftor combination regimen in people with cystic fibrosis homozygous for the F508del mutation: A double-blind, randomised, phase 3 trial. Lancet 2019, 394, 1940-1948. [CrossRef]

12. Griese, M.; Costa, S.; Linnemann, R.W.; Mall, M.A.; McKone, E.F.; Polineni, D.; Quon, B.S.; Ringshausen, F.C.; Taylor-Cousar, J.L.; Withers, N.J.; et al. Safety and Efficacy of Elexacaftor/Tezacaftor/Ivacaftor for 24 Weeks or Longer in People with Cystic Fibrosis and One or More F508del Alleles: Interim Results of an Open-Label Phase 3 Clinical Trial. Am. J. Respir. Crit. Care Med. 2021, 203, 381-385. [CrossRef] [PubMed]

13. Zemanick, E.T.; Taylor-Cousar, J.L.; Davies, J.; Gibson, R.L.; Mall, M.A.; McKone, E.F.; McNally, P.; Ramsey, B.W.; Rayment, J.H.; Rowe, S.M.; et al. A Phase 3 Open-Label Study of ELX/TEZ/IVA in Children 6 through 11 Years of Age with CF and at Least One F508del Allele. Am. J. Respir. Crit. Care Med. 2021. [CrossRef] [PubMed]

14. Riordan, J.R.; Rommens, J.M.; Kerem, B.; Alon, N.; Rozmahel, R.; Grzelczak, Z.; Zielenski, J.; Lok, S.; Plavsic, N.; Chou, J.L.; et al. Identification of the cystic fibrosis gene: Cloning and characterization of complementary DNA. Science 1989, 245, 1066-1073. [CrossRef]

15. Berschneider, H.M.; Knowles, M.R.; Azizkhan, R.G.; Boucher, R.C.; Tobey, N.A.; Orlando, R.C.; Powell, D.W. Altered intestinal chloride transport in cystic fibrosis. FASEB J. 1988, 2, 2625-2629. [CrossRef] [PubMed]

16. Taylor, C.J.; Baxter, P.S.; Hardcastle, J.; Hardcastle, P.T. Failure to induce secretion in jejunal biopsies from children with cystic fibrosis. Gut 1988, 29, 957-962. [CrossRef]

17. Hardcastle, J.; Hardcastle, P.T.; Taylor, C.J.; Goldhill, J. Failure of cholinergic stimulation to induce a secretory response from the rectal mucosa in cystic fibrosis. Gut 1991, 32, 1035-1039. [CrossRef]

18. Veeze, H.J.; Sinaasappel, M.; Bijman, J.; Bouquet, J.; de Jonge, H.R. Ion transport abnormalities in rectal suction biopsies from children with cystic fibrosis. Gastroenterology 1991, 101, 398-403. [CrossRef]

19. Veeze, H.J.; Halley, D.J.; Bijman, J.; de Jongste, J.C.; de Jonge, H.R.; Sinaasappel, M. Determinants of mild clinical symptoms in cystic fibrosis patients. Residual chloride secretion measured in rectal biopsies in relation to the genotype. J. Clin. Investig. 1994, 93, 461-466. [CrossRef]

20. Mall, M.; Bleich, M.; Greger, R.; Schreiber, R.; Kunzelmann, K. The amiloride-inhibitable $\mathrm{Na}^{+}$conductance is reduced by the cystic fibrosis transmembrane conductance regulator in normal but not in cystic fibrosis airways. J. Clin. Investig. 1998, $102,15-21$. [CrossRef]

21. Mall, M.; Bleich, M.; Schurlein, M.; Kuhr, J.; Seydewitz, H.H.; Brandis, M.; Greger, R.; Kunzelmann, K. Cholinergic ion secretion in human colon requires coactivation by cAMP. Am. J. Physiol. 1998, 275, G1274-G1281. [CrossRef]

22. Mall, M.; Bleich, M.; Kuehr, J.; Brandis, M.; Greger, R.; Kunzelmann, K. CFTR-mediated inhibition of epithelial Na ${ }^{+}$conductance in human colon is defective in cystic fibrosis. Am. J. Physiol. Gastrointest. Liver Physiol. 1999, 277, G709-G716. [CrossRef] [PubMed]

23. Mall, M.; Wissner, A.; Gonska, T.; Calenborn, D.; Kuehr, J.; Brandis, M.; Kunzelmann, K. Inhibition of amiloride-sensitive epithelial $\mathrm{Na}(+)$ absorption by extracellular nucleotides in human normal and cystic fibrosis airways. Am. J. Respir. Cell Mol. Biol. 2000, 23, 755-761. [CrossRef]

24. Mall, M.; Wissner, A.; Seydewitz, H.H.; Kuehr, J.; Brandis, M.; Greger, R.; Kunzelmann, K. Defective cholinergic Cl(-) secretion and detection of $\mathrm{K}(+)$ secretion in rectal biopsies from cystic fibrosis patients. Am. J. Physiol. Gastrointest. Liver Physiol. 2000, 278, G617-G624. [CrossRef] [PubMed]

25. Mall, M.; Wissner, A.; Seydewitz, H.H.; Hubner, M.; Kuehr, J.; Brandis, M.; Greger, R.; Kunzelmann, K. Effect of genistein on native epithelial tissue from normal individuals and CF patients and on ion channels expressed in Xenopus oocytes. Br. $J$. Pharmacol. 2000, 130, 1884-1892. [CrossRef] [PubMed]

26. Bronsveld, I.; Mekus, F.; Bijman, J.; Ballmann, M.; Greipel, J.; Hundrieser, J.; Halley, D.J.; Laabs, U.; Busche, R.; De Jonge, H.R.; et al. Residual chloride secretion in intestinal tissue of deltaF508 homozygous twins and siblings with cystic fibrosis. The European $\mathrm{CF}$ Twin and Sibling Study Consortium. Gastroenterology 2000, 119, 32-40. [CrossRef]

27. Kunzelmann, K.; Mall, M. Electrolyte transport in the mammalian colon: Mechanisms and implications for disease. Physiol. Rev. 2002, 82, 245-289. [CrossRef]

28. Hirtz, S.; Gonska, T.; Seydewitz, H.H.; Thomas, J.; Greiner, P.; Kuehr, J.; Brandis, M.; Eichler, I.; Rocha, H.; Lopes, A.I.; et al. CFTR Cl-channel function in native human colon correlates with the genotype and phenotype in cystic fibrosis. Gastroenterology 2004, 127, 1085-1095. [CrossRef]

29. Mall, M.; Hirtz, S.; Gonska, T.; Kunzelmann, K. Assessment of CFTR function in rectal biopsies for the diagnosis of cystic fibrosis. J. Cyst. Fibros. 2004, 3 (Suppl. 2), 165-169. [CrossRef]

30. Mall, M.; Kreda, S.M.; Mengos, A.; Jensen, T.J.; Hirtz, S.; Seydewitz, H.H.; Yankaskas, J.; Kunzelmann, K.; Riordan, J.R.; Boucher, R.C. The DeltaF508 mutation results in loss of CFTR function and mature protein in native human colon. Gastroenterology 2004, 126, 32-41. [CrossRef]

31. De Jonge, H.R.; Ballmann, M.; Veeze, H.; Bronsveld, I.; Stanke, F.; Tummler, B.; Sinaasappel, M. Ex vivo CF diagnosis by intestinal current measurements (ICM) in small aperture, circulating Ussing chambers. J. Cyst. Fibros. 2004, 3 (Suppl. 2), 159-163. [CrossRef]

32. Frizzell, R.A.; Rechkemmer, G.; Shoemaker, R.L. Altered regulation of airway epithelial cell chloride channels in cystic fibrosis. Science 1986, 233, 558-560. [CrossRef] [PubMed]

33. Knowles, M.R.; Clarke, L.L.; Boucher, R.C. Activation by extracellular nucleotides of chloride secretion in the airway epithelia of patients with cystic fibrosis. N. Engl. J. Med. 1991, 325, 533-538. [CrossRef] [PubMed] 
34. Anderson, M.P.; Welsh, M.J. Calcium and cAMP activate different chloride channels in the apical membrane of normal and cystic fibrosis epithelia. Proc. Natl. Acad. Sci. USA 1991, 88, 6003-6007. [CrossRef] [PubMed]

35. Gruber, A.D.; Schreur, K.D.; Ji, H.L.; Fuller, C.M.; Pauli, B.U. Molecular cloning and transmembrane structure of hCLCA2 from human lung, trachea, and mammary gland. Am. J. Physiol. 1999, 276, C1261-C1270. [CrossRef] [PubMed]

36. Clarke, L.; Boucher, R. Chloride secretory response to extracellular ATP in human normal and cystic fibrosis nasal epithelia. Am. J. Physiol. Cell Physiol. 1992, 263, C348-C356. [CrossRef]

37. Mall, M.; Gonska, T.; Thomas, J.; Schreiber, R.; Seydewitz, H.H.; Kuehr, J.; Brandis, M.; Kunzelmann, K. Modulation of $\mathrm{Ca}^{2+}$-activated $\mathrm{Cl}^{-}$secretion by basolateral $\mathrm{K}^{+}$channels in human normal and cystic fibrosis airway epithelia. Pediatr. Res. 2003, 53, 608-618. [CrossRef]

38. Caputo, A.; Caci, E.; Ferrera, L.; Pedemonte, N.; Barsanti, C.; Sondo, E.; Pfeffer, U.; Ravazzolo, R.; Zegarra-Moran, O.; Galietta, L.J. TMEM16A, a membrane protein associated with calcium-dependent chloride channel activity. Science 2008, 322, 590-594 [CrossRef] [PubMed]

39. Salomon, J.J.; Albrecht, T.; Graeber, S.Y.; Scheuermann, H.; Butz, S.; Schatterny, J.; Mairbaurl, H.; Baumann, I.; Mall, M.A. Chronic rhinosinusitis with nasal polyps is associated with impaired TMEM16A-mediated epithelial chloride secretion. J. Allergy Clin. Immunol. 2021. [CrossRef]

40. Derichs, N.; Sanz, J.; Von Kanel, T.; Stolpe, C.; Zapf, A.; Tummler, B.; Gallati, S.; Ballmann, M. Intestinal current measurement for diagnostic classification of patients with questionable cystic fibrosis: Validation and reference data. Thorax 2010, 65, 594-599. [CrossRef] [PubMed]

41. De Boeck, K.; Kent, L.; Davies, J.; Derichs, N.; Amaral, M.; Rowe, S.M.; Middleton, P.; de Jonge, H.; Bronsveld, I.; Wilschanski, M.; et al. CFTR biomarkers: Time for promotion to surrogate end-point. Eur. Respir. J. 2013, 41, 203-216. [CrossRef] [PubMed]

42. Wilschanski, M.; Yaakov, Y.; Omari, I.; Zaman, M.; Martin, C.R.; Cohen-Cymberknoh, M.; Shoseyov, D.; Kerem, E.; Dasilva, D.; Sheth, S.; et al. Comparison of Nasal Potential Difference and Intestinal Current Measurements as Surrogate Markers for CFTR Function. J. Pediatr. Gastroenterol. Nutr. 2016, 63, e92-e97. [CrossRef] [PubMed]

43. Roth, E.K.; Hirtz, S.; Duerr, J.; Wenning, D.; Eichler, I.; Seydewitz, H.H.; Amaral, M.D.; Mall, M.A. The K ${ }^{+}$channel opener 1-EBIO potentiates residual function of mutant CFTR in rectal biopsies from cystic fibrosis patients. PLoS ONE 2011, 6, e24445. [CrossRef] [PubMed]

44. Graeber, S.Y.; Hug, M.J.; Sommerburg, O.; Hirtz, S.; Hentschel, J.; Heinzmann, A.; Dopfer, C.; Schulz, A.; Mainz, J.G.; Tummler, B.; et al. Intestinal Current Measurements Detect Activation of Mutant CFTR in Patients with Cystic Fibrosis with the G551D Mutation Treated with Ivacaftor. Am. J. Respir. Crit. Care Med. 2015, 192, 1252-1255. [CrossRef]

45. Graeber, S.Y.; Dopfer, C.; Naehrlich, L.; Gyulumyan, L.; Scheuermann, H.; Hirtz, S.; Wege, S.; Mairbaurl, H.; Dorda, M.; Hyde, R.; et al. Effects of Lumacaftor-Ivacaftor Therapy on Cystic Fibrosis Transmembrane Conductance Regulator Function in Phe508del Homozygous Patients with Cystic Fibrosis. Am. J. Respir. Crit. Care Med. 2018, 197, 1433-1442. [CrossRef]

46. Masson, A.; Schneider-Futschik, E.K.; Baatallah, N.; Nguyen-Khoa, T.; Girodon, E.; Hatton, A.; Flament, T.; Le Bourgeois, M.; Chedevergne, F.; Bailly, C.; et al. Predictive factors for lumacaftor/ivacaftor clinical response. J. Cyst. Fibros. 2019, 18, 368-374. [CrossRef] [PubMed]

47. Sousa, M.; Servidoni, M.F.; Vinagre, A.M.; Ramalho, A.S.; Bonadia, L.C.; Felicio, V.; Ribeiro, M.A.; Uliyakina, I.; Marson, F.A.; $\mathrm{Kmit}$, A.; et al. Measurements of CFTR-mediated $\mathrm{Cl}^{-}$secretion in human rectal biopsies constitute a robust biomarker for Cystic Fibrosis diagnosis and prognosis. PLoS ONE 2012, 7, e47708. [CrossRef] [PubMed]

48. Beekman, J.M.; Sermet-Gaudelus, I.; de Boeck, K.; Gonska, T.; Derichs, N.; Mall, M.A.; Mehta, A.; Martin, U.; Drumm, M.; Amaral, M.D. CFTR functional measurements in human models for diagnosis, prognosis and personalized therapy: Report on the pre-conference meeting to the 11th ECFS Basic Science Conference, Malta, 26-29 March 2014. J. Cyst. Fibros. 2014, 13, 363-372. [CrossRef]

49. Cohen-Cymberknoh, M.; Yaakov, Y.; Shoseyov, D.; Shteyer, E.; Schachar, E.; Rivlin, J.; Bentur, L.; Picard, E.; Aviram, M.; Israeli, E. Evaluation of the intestinal current measurement method as a diagnostic test for cystic fibrosis. Pediatr. Pulmonol. 2013, 48, 229-235. [CrossRef] [PubMed]

50. Clancy, J.P.; Szczesniak, R.D.; Ashlock, M.A.; Ernst, S.E.; Fan, L.; Hornick, D.B.; Karp, P.H.; Khan, U.; Lymp, J.; Ostmann, A.J.; et al. Multicenter intestinal current measurements in rectal biopsies from CF and non-CF subjects to monitor CFTR function. PLoS ONE 2013, 8, e73905. [CrossRef]

51. Farinha, C.M.; King-Underwood, J.; Sousa, M.; Correia, A.R.; Henriques, B.J.; Roxo-Rosa, M.; Da Paula, A.C.; Williams, J.; Hirst, S.; Gomes, C.M.; et al. Revertants, low temperature, and correctors reveal the mechanism of F508del-CFTR rescue by VX-809 and suggest multiple agents for full correction. Chem. Biol. 2013, 20, 943-955. [CrossRef] [PubMed]

52. Farrell, P.M.; White, T.B.; Ren, C.L.; Hempstead, S.E.; Accurso, F.; Derichs, N.; Howenstine, M.; McColley, S.A.; Rock, M.; Rosenfeld, M.; et al. Diagnosis of Cystic Fibrosis: Consensus Guidelines from the Cystic Fibrosis Foundation. J. Pediatr. 2017, 181S, S4-S15.e11. [CrossRef] [PubMed]

53. Minso, R.; Schulz, A.; Dopfer, C.; Alfeis, N.; Barneveld, A.V.; Makartian-Gyulumyan, L.; Hansen, G.; Junge, S.; Muller, C.; Ringshausen, F.C.C.; et al. Intestinal current measurement and nasal potential difference to make a diagnosis of cases with inconclusive CFTR genetics and sweat test. BMJ Open Respir. Res. 2020, 7. [CrossRef] 
54. Silva, I.A.L.; Duarte, A.; Marson, F.A.L.; Centeio, R.; Doušová, T.; Kunzelmann, K.; Amaral, M.D. Assessment of Distinct Electrophysiological Parameters in Rectal Biopsies for the Choice of the Best Diagnosis/Prognosis Biomarkers for Cystic Fibrosis. Front. Physiol. 2020, 11, 604580. [CrossRef]

55. Sermet-Gaudelus, I.; Brouard, J.; Audrézet, M.P.; Couderc Kohen, L.; Weiss, L.; Wizla, N.; Vrielynck, S.; Llerena, K.; Le Bourgeois, M.; Deneuville, E.; et al. Guidelines for the clinical management and follow-up of infants with inconclusive cystic fibrosis diagnosis through newborn screening. Arch. Pediatr. 2017, 24, e1-e14. [CrossRef]

56. Barben, J.; Castellani, C.; Munck, A.; Davies, J.C.; de Winter-de Groot, K.M.; Gartner, S.; Kashirskaya, N.; Linnane, B.; Mayell, S.J.; McColley, S.; et al. Updated guidance on the management of children with cystic fibrosis transmembrane conductance regulator-related metabolic syndrome/cystic fibrosis screen positive, inconclusive diagnosis (CRMS/CFSPID). J. Cyst. Fibros. 2020. [CrossRef] [PubMed]

57. Sommerburg, O.; Lindner, M.; Muckenthaler, M.; Kohlmueller, D.; Leible, S.; Feneberg, R.; Kulozik, A.E.; Mall, M.A.; Hoffmann, G.F. Initial evaluation of a biochemical cystic fibrosis newborn screening by sequential analysis of immunoreactive trypsinogen and pancreatitis-associated protein (IRT/PAP) as a strategy that does not involve DNA testing in a Northern European population. J. Inherit. Metab. Dis. 2010, 33, S263-S271. [CrossRef] [PubMed]

58. Sommerburg, O.; Krulisova, V.; Hammermann, J.; Lindner, M.; Stahl, M.; Muckenthaler, M.; Kohlmueller, D.; Happich, M.; Kulozik, A.E.; Votava, F.; et al. Comparison of different IRT-PAP protocols to screen newborns for cystic fibrosis in three central European populations. J. Cyst. Fibros. 2014, 13, 15-23. [CrossRef] [PubMed]

59. Sato, T.; Clevers, H. Growing self-organizing mini-guts from a single intestinal stem cell: Mechanism and applications. Science 2013, 340, 1190-1194. [CrossRef]

60. Dekkers, J.F.; Wiegerinck, C.L.; de Jonge, H.R.; Bronsveld, I.; Janssens, H.M.; de Winter-de Groot, K.M.; Brandsma, A.M.; de Jong, N.W.M.; Bijvelds, M.J.C.; Scholte, B.J.; et al. A functional CFTR assay using primary cystic fibrosis intestinal organoids. Nat. Med. 2013, 19, 939-945. [CrossRef]

61. Awatade, N.T.; Uliyakina, I.; Farinha, C.M.; Clarke, L.A.; Mendes, K.; Solé, A.; Pastor, J.; Ramos, M.M.; Amaral, M.D. Measurements of Functional Responses in Human Primary Lung Cells as a Basis for Personalized Therapy for Cystic Fibrosis. EBioMedicine 2015, 2, 147-153. [CrossRef]

62. Pranke, I.M.; Hatton, A.; Simonin, J.; Jais, J.P.; Le Pimpec-Barthes, F.; Carsin, A.; Bonnette, P.; Fayon, M.; Stremler-Le Bel, N.; Grenet, D. Correction of CFTR function in nasal epithelial cells from cystic fibrosis patients predicts improvement of respiratory function by CFTR modulators. Sci. Rep. 2017, 7, 7375. [CrossRef] [PubMed]

63. Mutyam, V.; Libby, E.F.; Peng, N.; Hadjiliadis, D.; Bonk, M.; Solomon, G.M.; Rowe, S.M. Therapeutic benefit observed with the CFTR potentiator, ivacaftor, in a CF patient homozygous for the W1282X CFTR nonsense mutation. J. Cyst. Fibros. 2017, 16, 24-29. [CrossRef]

64. Pranke, I.; Hatton, A.; Masson, A.; Flament, T.; Le Bourgeois, M.; Chedevergne, F.; Bailly, C.; Urbach, V.; Hinzpeter, A.; Edelman, A.; et al. Might Brushed Nasal Cells Be a Surrogate for CFTR Modulator Clinical Response? Am. J. Respir. Crit. Care Med. 2019, 199, 123-126. [CrossRef] [PubMed]

65. Amato, F.; Scudieri, P.; Musante, I.; Tomati, V.; Caci, E.; Comegna, M.; Maietta, S.; Manzoni, F.; Di Lullo, A.M.; De Wachter, E.; et al. Two CFTR mutations within codon 970 differently impact on the chloride channel functionality. Hum. Mutat. 2019, 40, 742-748. [CrossRef] [PubMed]

66. Laselva, O.; Moraes, T.J.; He, G.; Bartlett, C.; Szàrics, I.; Ouyang, H.; Gunawardena, T.N.A.; Strug, L.; Bear, C.E.; Gonska, T. The CFTR Mutation c.3453G > C (D1152H) Confers an Anion Selectivity Defect in Primary Airway Tissue That Can Be Rescued by Ivacaftor. J. Pers. Med. 2020, 10, 40. [CrossRef] [PubMed]

67. Laselva, O.; McCormack, J.; Bartlett, C.; Ip, W.; Gunawardena, T.N.A.; Ouyang, H.; Eckford, P.D.W.; Gonska, T.; Moraes, T.J.; Bear, C.E. Preclinical Studies of a Rare CF-Causing Mutation in the Second Nucleotide Binding Domain (c.3700A>G) Show Robust Functional Rescue in Primary Nasal Cultures by Novel CFTR Modulators. J. Pers. Med. 2020, 10, 209. [CrossRef] [PubMed]

68. Phuan, P.W.; Haggie, P.M.; Tan, J.A.; Rivera, A.A.; Finkbeiner, W.E.; Nielson, D.W.; Thomas, M.M.; Janahi, I.A.; Verkman, A.S. CFTR modulator therapy for cystic fibrosis caused by the rare c.3700A $>\mathrm{G}$ mutation. J. Cyst. Fibros. 2020. [CrossRef]

69. Veit, G.; Roldan, A.; Hancock, M.A.; Da Fonte, D.F.; Xu, H.; Hussein, M.; Frenkiel, S.; Matouk, E.; Velkov, T.; Lukacs, G.L. Allosteric folding correction of F508del and rare CFTR mutants by elexacaftor-tezacaftor-ivacaftor (Trikafta) combination. JCI Insight 2020, 5 . [CrossRef]

70. Laselva, O.; Eckford, P.D.W.; Bartlett, C.; Ouyang, H.; Gunawardena, T.N.A.; Gonska, T.; Moraes, T.J.; Bear, C.E. Functional rescue of c.3846G $>\mathrm{A}(\mathrm{W} 1282 \mathrm{X})$ in patient-derived nasal cultures achieved by inhibition of nonsense mediated decay and protein modulators with complementary mechanisms of action. J. Cyst. Fibros. 2020, 19, 717-727. [CrossRef]

71. Molinski, S.V.; Ahmadi, S.; Ip, W.; Ouyang, H.; Villella, A.; Miller, J.P.; Lee, P.S.; Kulleperuma, K.; Du, K.; Di Paola, M.; et al. Orkambi ${ }^{\circledR}$ and amplifier co-therapy improves function from a rare CFTR mutation in gene-edited cells and patient tissue. EMBO Mol. Med. 2017, 9, 1224-1243. [CrossRef]

72. Veit, G.; Xu, H.; Dreano, E.; Avramescu, R.G.; Bagdany, M.; Beitel, L.K.; Roldan, A.; Hancock, M.A.; Lay, C.; Li, W.; et al. Structure-guided combination therapy to potently improve the function of mutant CFTRs. Nat. Med. 2018, 24, 1732-1742. [CrossRef] 
73. Laselva, O.; Bartlett, C.; Gunawardena, T.N.A.; Ouyang, H.; Eckford, P.D.W.; Moraes, T.J.; Bear, C.E.; Gonska, T. Rescue of multiple class II CFTR mutations by elexacaftor + tezacaftor + ivacaftor mediated in part by the dual activities of Elexacaftor as both corrector and potentiator. Eur. Respir. J. 2020. [CrossRef]

74. Laselva, O.; Bartlett, C.; Popa, A.; Ouyang, H.; Gunawardena, T.N.A.; Gonska, T.; Moraes, T.J.; Bear, C.E. Emerging preclinical modulators developed for F508del-CFTR have the potential to be effective for ORKAMBI resistant processing mutants. J. Cyst. Fibros. 2021, 20, 106-119. [CrossRef] [PubMed]

75. de Winter-de Groot, K.M.; Janssens, H.M.; van Uum, R.T.; Dekkers, J.F.; Berkers, G.; Vonk, A.; Kruisselbrink, E.; Oppelaar, H.; Vries, R.; Clevers, H.; et al. Stratifying infants with cystic fibrosis for disease severity using intestinal organoid swelling as a biomarker of CFTR function. Eur. Respir. J. 2018, 52. [CrossRef] [PubMed]

76. Graeber, S.Y.; van Mourik, P.; Vonk, A.M.; Kruisselbrink, E.; Hirtz, S.; van der Ent, C.K.; Mall, M.A.; Beekman, J.M. Comparison of Organoid Swelling and In Vivo Biomarkers of CFTR Function to Determine Effects of Lumacaftor-Ivacaftor in Patients with Cystic Fibrosis Homozygous for the F508del Mutation. Am. J. Respir. Crit. Care Med. 2020, 202, 1589-1592. [CrossRef] [PubMed]

77. Berkers, G.; van der Meer, R.; Heijerman, H.; Beekman, J.M.; Boj, S.F.; Vries, R.G.J.; van Mourik, P.; Doyle, J.R.; Audhya, P.; Yuan, Z.J.; et al. Lumacaftor/ivacaftor in people with cystic fibrosis with an A455E-CFTR mutation. J. Cyst. Fibros. 2020. [CrossRef]

78. Aalbers, B.L.; Hofland, R.W.; Bronsveld, I.; de Winter-de Groot, K.M.; Arets, H.G.M.; de Kiviet, A.C.; van Oirschot-van de Ven, M.M.M.; Kruijswijk, M.A.; Schotman, S.; Michel, S.; et al. Females with cystic fibrosis have a larger decrease in sweat chloride in response to lumacaftor/ivacaftor compared to males. J. Cyst. Fibros. 2021, 20, e7-e11. [CrossRef] [PubMed]

79. Graeber, S.Y.; Boutin, S.; Wielputz, M.O.; Joachim, C.; Frey, D.L.; Wege, S.; Sommerburg, O.; Kauczor, H.U.; Stahl, M.; Dalpke, A.H.; et al. Effects of Lumacaftor-Ivacaftor on Lung Clearance Index, Magnetic Resonance Imaging and Airway Microbiome in Phe508del Homozygous Patients with Cystic Fibrosis. Ann. Am. Thorac. Soc. 2021. [CrossRef]

80. Bagheri-Hanson, A.; Nedwed, S.; Rueckes-Nilges, C.; Naehrlich, L. Intestinal current measurement versus nasal potential difference measurements for diagnosis of cystic fibrosis: A case-control study. BMC Pulm. Med. 2014, 14, 156. [CrossRef] 